\title{
Regional Anaesthetic Techniques and Their Implications During the COVID Pandemic
}

\author{
Raafay Mehmood ${ }^{1}$ (1) $\cdot$ Ainsley John McGuire ${ }^{2} \cdot$ Zainab Mansoor $^{1} \cdot$ Adam Benjamin Fink $^{1} \cdot$ Gabriel Atanasov $^{1}$
}

Accepted: 27 July 2021 / Published online: 21 September 2021

(c) The Author(s), under exclusive licence to Springer Nature Switzerland AG 2021

\begin{abstract}
The current pandemic has highlighted the need to protect both patients and medical staff. The increased use of regional anaesthesia as a primary anaesthetic modality for operations and other invasive procedures has limited the number of aerosolgenerating procedures performed during general anaesthesia. Its use is further characterized by decreases in postoperative pain and length of hospitalization. This article provides an overview of regional anaesthetic techniques (peripheral nerve locks, epidural and spinal anaesthesia) and their uses during the COVID pandemic.
\end{abstract}

Keywords Regional anaesthesia $\cdot$ Peripheral nerve block $\cdot$ Spinal anaesthesia $\cdot$ Epidural anaesthesia

\section{Introduction}

The COVID-19 pandemic is a significant burden on healthcare systems globally. As anaesthesiologists are front line workers in surgical management, they are often exposed to patients' respiratory secretions, placing themselves at further risk of viral transmission. It becomes important to minimize the utilization of aerosol-generating procedures (AGPs) often performed during general anaesthesia (GA) [1]. These include bag-mask ventilation, bronchoscopy, bronchoalveolar lavage, endotracheal intubation, extubation and open airway suctioning [2]. Regional anaesthesia (RA) can be used to avoid airway manipulation, and it has become a common modality in surgical procedures during the pandemic [3].

RA involves the reversible numbing of a specific body part in order to prevent any sensation of pain [4]. It can further be divided into peripheral nerve blocks and neuraxial anaesthesia (spinal and epidural anaesthesia). Although GA can be used in a variety of surgeries, the use of RA provides further benefits in addition to reduced airway management including the following: decreased postoperative nausea; decreased stay in the post-anaesthesia care unit (PACU);

Raafay Mehmood

Raafay95@hotmail.com

1 First Faculty of Medicine, Charles University, Prague, Czech Republic

2 Faculty of Health Sciences, University of Northern British Columbia, Prince George, Canada lower risk of respiratory and gastrointestinal complications; and fewer opioid related side effects (pruritis and respiratory depression) [5]. Furthermore, RA is commonly used to reduce acute postoperative pain, which is reported by more than $80 \%$ of patients undergoing surgery [6]. Treatment of postoperative pain results in improved clinical outcomes, as it has been investigated that poor acute pain control can lead to chronic devastating pain [7]. This is commonly seen in orthopaedic surgery, which is highly associated with severe postoperative pain, where a suitable pain management protocol to improve the patient's range of movement is vital for successful rehabilitation and early return to daily activities $[8,9]$. The purpose of this manuscript is to review the literature and evaluate data pertaining to the advantages and disadvantages of RA techniques.

\section{Peripheral Nerve Blocks}

A peripheral nerve block (PNB) involves the use of a local anaesthetic injection in the proximity of a nerve or peripheral nerve bundle, which results in the inhibition of the excitatory action potential, responsible for transmitting a nociceptive stimulus along the various nerve fibres towards the central nervous system [10]. They can be used independently as the only anaesthetic, or used as a supplement to GA. There are different types of nerve blocks such as specific nerve blocks, brachial plexus blocks and paravertebral blocks as well as a single injection and continuous 
nerve blocks. They are commonly used in surgeries involving the following: the extremities, elderly patients [11] and in certain major orthopaedic surgeries such as shoulder and hip replacement [10]. Additionally, PNBs are often used to modulate the pain response postoperatively with the use of single shot or continuous nerve blocks [12-15]. Their use is contraindicated in cases of allergy to the local anaesthetic agent, in the presence of certain illnesses such as epilepsy, porphyria (lidocaine), chronic lung disease, severe liver disease, peripheral neuropathies, haemostasis and coagulation disorders, as well as patient refusal and infection at the puncture site [16].

PNBs are carried out in 3 steps: preparation, administration and recovery. The first step can be considered the most crucial, as it requires a thorough patient history and preoperative assessment regarding the PNB technique and anaesthetic risk. During this step, patient consent is received, confirmation that the patient is in a fasting state as well as confirmation that the patient does not have any contraindications [10]. Following this step, the PNB is administered in a process that requires patient positioning, anatomical landmark identification and puncture [17]. During the puncture, a motor response is evoked by a nerve stimulator and/or ultrasound to localize the nerve, and after this, the injection of anaesthetic can begin. This consists of a slow injection of anaesthetic with the subsequent loss of the motor response. Once the anaesthesia has been administered, there is an inhibition of proprioceptive transmission, resulting in the regional block of nerve transmission from the target nerve. It becomes extremely important to monitor the patient for signs of both cardiotoxicity and neurotoxicity. The last stage begins upon completion of the procedure and involves the patient transfer to the PACU. There, the patient is continuously monitored until they are deemed to have no complications from anaesthesia and surgery until their subsequent discharge [10].

PNBs can be implemented in a wide variety of cases, for example, treatment for primary headache disorders, where the greater and lesser occipital nerves are commonly used, although sometimes several branches of the trigeminal nerve can also be used [18]. Continuous PNBs are routinely used in the peri- and postoperative period; however, their use in the treatment of chronic pain in cancer, complex regional pain syndrome and phantom limb pain has also been welldocumented [19]. PNBs, in addition to their many different indications, offer a range of benefits which include decrease of baseline pain, reduction of any additional analgesic requirements and reduced postoperative inflammation and can be utilized in the treatment of sleep disorders. However, the safety and success of PNBs are dependent on the correct technique for delivery of the local anaesthetic as well as the correct dose. Failure to do so can result in potential, although rare, complications such as systemic toxicity, infection, nerve damage and bleeding [20,21].

\section{Epidural Anaesthesia}

Epidural anaesthesia (EA), is not only a subtype of neuraxial anaesthesia, but is also a type of regional anaesthesia. It involves the insertion of a hollow needle, alongside a small and flexible catheter, into the epidural space-which contains fat, blood vessels, lymphatics, and the spinal nerves exiting the spinal cord [22]. Furthermore, it can be performed in the cervical, lumbar or thoracic regions of the spine as well as the sacral hiatus [23]. Opioids, anaesthetic and analgesic agents can be delivered through the catheter to achieve different purposes. EA can be provided alone or in conjunction with general anaesthesia for postoperative pain modulation. In terms of the distribution of an epidural neural block, significant factors include the mass of local anaesthetic, the site of injection and patient variables in the determination of the extent of the sensory, sympathetic, and motor neural blockade [24]. Height, weight and BMI do not have a clinically significant impact on the spread of epidural block. However, age does have an effect as a given dose of local anaesthetic agent will spread farther in older patients when compared to younger patients [25].

EA is commonly used in surgical settings to reduce perioperative stress and thereby improve postoperative outcomes; meta-studies have indicated a significant reduction in perioperative cardiac morbidity, pulmonary infections, pulmonary embolism, acute renal failure and blood loss [26]. EA is used frequently in labour pain relief, surgeries of the abdominal, intra-thorax or lower extremities and used to modulate pain postoperatively [25]. In comparison to spinal anaesthesia (SA), EA requires greater dosages of local anaesthetic and takes longer to implement. However, once the catheter is in place, anaesthetic agents can be repeatedly administered, and their effects can be prolonged to match the surgery. The contraindications of EA are similar to those of SA due to both being types of neuraxial anaesthesia. Their use is contraindicated in cases such as patient refusal, compromised sterility of medical instruments, coagulopathies, infection at the site of injection, increased intracranial pressure, severe aortic stenosis, severe mitral stenosis, an allergy to local anaesthetics and severe uncorrected anaemia. Relative contraindications of EA include sepsis, severe spinal deformities, an uncooperative patient, demyelinating lesions, pre-existing neurological defects and stenotic valvular heart lesions [27].

EA is carried out in a series of consecutive steps. The first step is patient preparation, entailing the assembly of equipment needed, positioning of the patient (which can be either sitting or lateral dependant on the procedure), current clinical situation (hip fractures, emergency C-section) 
and the patient and provider. Patients with a BMI under 25 reported being more comfortable in the lateral position, whereas patients with a BMI greater than 30 reported being more comfortable sitting [28]. Following this step, anatomical landmarks are palpated (such as the spinous processes thus aiding in identifying the midline), and puncture can commence. Before puncture can occur, an approach must be decided either midline or paramedian. LEA can be performed with either; however, TEA is performed more easily using the paramedian approach. This is due to the thoracic spine having a lesser degree of flexibility compared to the lumbar spine and the steeper slope of the thoracic spinous processes. Epidural needles usually have a curved tip for guidance into the epidural space; in comparison to spinal needles, they are typically larger. This feature improves the tactile feel as the needle passes through the ligamentum flavum and enters into the epidural space.

After the puncture, identification of the epidural space can be done either through the hanging drop or loss of resistance technique. The hanging drop technique relies on negative air pressure within the epidural space. On the hub of the needle, a drop of saline is placed. Upon entry into the epidural space, the drop will then be pulled into the needle [29]. This method is more reliable for TEA than LEA. *The loss of resistance technique is universally used: if the tip of the needle is within the ligamentum flavum resistance will be encountered, a transition occurs when the needle tip enters the epidural space, as a loss of resistance is perceived [30]. For this method, air or saline can be used in the loss of resistance syringe. There is discussion as to which is more advantageous, although a review conducted in 2014 determined no benefits of saline versus air in terms of locating the epidural space [31]. However, another review highlighted that the use of saline provides analgesic superiority as well as decreasing morbidity, while also reporting fewer occurrences of complications such as incomplete analgesia, pneumocephalus, nerve root compression, subcutaneous emphysema and venous air embolism [32]. After identification of the epidural space, the catheter can be inserted into the epidural space, and the needle can be removed. Placement of a catheter allows for EA to be provided for as long as needed. Depending on the clinical scenario, catheters with a single orifice or multi-orifice can be used, with the latter allowing for a greater distribution of the anaesthetic agent. In labouring women, they are associated with better analgesia and a more extensive block [33].

\section{Spinal Anaesthesia}

Spinal anaesthesia is a subtype of neuraxial anaesthesia, where local anaesthetic is injected $2-3 \mathrm{~mm}$ beyond the epidural space, intrathecally, blocking all motor and sensory function below the upper level of the block [34]. This initially blocks small unmyelinated sympathetic fibres, followed by the blockage of both myelinated sensory and motor fibres. Although there are no absolute indications for SA, its use is dependent on patient, surgeon and anaesthesiologists' preferences. It is, however, commonly indicated for surgeries of the lower abdomen, perineal and lower extremities [35]. It is almost exclusively done in the lumbar region, below the termination of the spinal cord. It can be used for upper abdominal surgeries, but the impact on breathing at that level calls for the use of GA.

A spinal block consists of a single injection with lasting effects up to $3 \mathrm{~h}$ after administration, hence making it unsuitable for lengthy or unpredictable operations [25]. Additionally, the motor nerve block generated is sufficient to use when preforming major surgeries such as hip or knee replacements. However, continuous SA with the use of a catheter can be used to provide a prolonged duration of the spinal block, but it requires a dural puncture with a large gauge needle. These needles are associated with high incidences of headaches [36]. Yet, the smaller the needle, the lower the risk for post-dural puncture headache. Additionally, continuous spinal catheters have the potential to be mistaken as an epidural catheter, which can lead to dangerous levels of the anaesthetic in the subarachnoid space, if a large epidural dose is administered. If unrecognized, this can lead to respiratory and cardiac arrest [37]. This is often the reason for high block in labouring women during an attempted epidural [38, 39].

SA provides an alternative to general anaesthesia for several emergent situations such as emergency caesarean sections, appendectomies and many elective surgeries, especially during this pandemic [40]. In 2003, an SA technique was developed to address the need for SA in emergency obstetrics, known as rapid sequence SA. This may now be indicated, as most anaesthetic personnel are already wearing the required personal protective equipment (PPE) regardless of COVID positivity [41]. This technique addresses the time and preparation required to perform classical SA, reducing the requirements to a pair of sterile gloves, and is performed using a single puncture without palpating anatomical landmarks [42]. A second puncture is utilized if necessary, before immediately transitioning to general anaesthesia in case of failure. A retrospective study carried out in Wuhan (the epicentre of the pandemic in early 2020) [43] found that only $2.7 \%$ of anaesthesiologists who encountered PCR positive patients while preforming SA then developed subsequent COVID infections, providing that they were wearing level 3 PPE. This highlights the safety in minimizing aerosol-generating procedures and using techniques such as SA instead of GA, where the case permits.

It has been hypothesized for some time now that SA may produce better patient outcomes in comparison to the use of 
a GA for a given surgical procedure. A meta-analysis performed in 2018 found that patients who received SA prior to total hip arthroplasties required shorter hospitalizations postoperation and reported significantly lower levels of nausea in comparison to patients who underwent GA [44]. Conversely, a randomized control trial from the same year comparing the outcomes of patients who underwent foot and ankle surgery found that patients who received GA were discharged earlier than those who received SA, while there were no differences regarding reported nausea, opioid use, headaches or back pain [45]. Additionally, a 2018 study comparing the perioperative and postoperative outcomes of patients who underwent laparoscopic appendectomies found that those who had the procedure performed under SA suffered less surgical field pain and had a longer postoperative pain free period, while also reporting less postoperative nausea and vomiting [46].

\section{Benefits Over General Anaesthesia}

RA carries a risk profile for possible complications that can be variable depending on the clinical setting and purpose for which it is applied. This makes any sort of definitive answer on its overall safety impossible at this time, given the lack of studies extensively examining all the diverse forms these procedures can take, and the even more diverse populations they are performed on [47]. Additionally, research that examines the rates of complications and overall outcomes associated with RA alone versus GA alone is not yet fully developed. The uncertainty this creates in making comparisons between RA and GA is compounded by the fact that they are often more commonly indicated for different kinds of surgery [48]. However, there is a growing body of evidence suggesting that the use of RA, in combination with GA or alone, decreases the rates of some complications and improves some postoperative outcomes compared to procedures employing only general anaesthesia [49].

One major grouping of complications where the benefits of RA are supported in a number of surgical settings are those involving the cardiovascular system, including, but not limited to, conditions such as deep venous thrombosis, pulmonary embolism and cardiac events. The deep sympathetic blockade provided by the use of neuraxial anaesthesia in intrathoracic and intra-abdominal surgeries results in improved lower extremity blood flow, lowered incidence of hypercoagulability and a reduced cardiac effort [50]. Furthermore, the use of postoperative epidural anaesthesia offers superior analgesia, continuous low-dose local anaesthetic effects as well as avoidance of systemic opioids. This in turn results in improved bowel mobility; improved coughing and breathing; and earlier ambulation, further reducing the risk of complications related to circulation and coagulation in the extremities [50,51].

RA causes fewer incidences of postoperative side effects increasing the length of hospital stay. Postoperative nausea and vomiting can lead to not only longer post-anaesthesia care unit stays but also delayed day surgery unit discharge and unplanned hospital admission. The mechanism for the reduction of this side effect with RA is likely to be multifactorial, yet probably related to the avoidance of systemic opioids and other sedatives that are known to disrupt bowel function [50, 52]. Reduced quality of sleep is another side effect related to the length of stay. Shorter duration of sleep, fragmented sleep patterns and reduced REM sleep have been associated with increased nociception. This can impact subsequent sleep and enhanced recovery programs. Furthermore, a later rebound in REM sleep has been associated with myocardial infarction, stroke and hemodynamic instability. The use of neuraxial anaesthesia intraoperatively, as well as in postoperative pain management, has been associated with improved sleep outcomes post-surgery, including a decrease in obstructive sleep apnoea, and likely benefits related to the reduced use of systemic opioids and sedatives [50, 53]. The severity of acute pain in the postoperative period has also been associated with increased duration of hospitalization, as well as being a factor in the likelihood of chronic postoperative pain. Epidural analgesia (regardless of the agent used, the type of surgery or the method of pain assessment) has been shown to provide superior analgesia to parenteral opioids [54].

RA also shows benefits in terms of reducing pulmonary side effects and postoperative respiratory function, with virtually no side effects in healthy people when administered correctly, and positive benefits for risk of complications in patients with pre-existing conditions like COPD and asthma $[50,55]$. The mechanism for these benefits is largely to do with avoidance of the relatively numerous respiratory side effects stemming from general anaesthesia's effects on consciousness; mode of ventilation; posture during the intraoperative period; as well as the actions of the anaesthetic agents and drugs used during anaesthesia on respiratory smooth muscles and secretions $[56,57]$.

\section{Implications During the COVID Pandemic}

During the pandemic, organisations such as The American Society of Regional Anesthesia and Pain Medicine and European Society of Regional Anaesthesia and Pain Therapy have highly encouraged the preferential use of regional anaesthesia, whenever possible, to minimize AGPs [40]. It has also become essential to pre-plan when performing RA, as the anaesthesiologist must be prepared for a failed block and conversion to GA, which increases the risk of 
COVID transmission. The advantages RA holds in minimizing respiratory transmission disappear in the case of a COVID positive patient undergoing unplanned conversion to GA. Therefore, it is adamant that the anaesthesiologist plans accordingly to whether the whole operation can be carried out under RA; otherwise, it may be beneficial to start with GA rather than preparing for low-probability RA. Likewise, GA will be preferred in critically unwell intubated patients.

Severe COVID disease has the propensity to cause thrombocytopenia, which is a common contraindication for RA [58]. Anaesthesiologists screen both COVID positive and negative patients during a thorough pre-operative evaluation to deem their fitness for RA. In COVID positive patients, a review of a full blood count, percent saturation of haemoglobin with oxygen $\left(\mathrm{SpO}_{2}>93 \%\right)$ and a suitable international normalised ratio $(<1.5)$ is required at minimum [59]. Additionally, a COVID infection can lead to a reduced respiratory reserve, which can be problematic for patients undergoing shoulder surgery, since the brachial plexus and interscalene block frequently cause phrenic nerve palsy [60]. Anaesthetic management of these patients requires limiting both the dose and volume of the local anaesthetic or alternatively performing suprascapular and axillary blocks to avoid any disturbance of the phrenic nerve.

As previously mentioned, the use of RA causes fewer pulmonary side effects compared to GA. A reduction in respiratory side effects and increased opportunity to forgo mechanical ventilation would likely contribute to a reduced risk of viral transmission associated with invasive respiratory intervention, shorter postoperative hospitalization and reduced use of respiratory support equipment urgently required in hospitals with large numbers of patients suffering the severe respiratory symptoms associated with COVID-19 $[56,61]$. However, RA techniques are commonly carried out with intraoperative sedatives, which can also cause some respiratory depression [40]. In COVID positive patients, sedatives are avoided or used minimally.

RA's role in reducing postoperative side effects and resultant hospital stay is especially significant in the present settings of the COVID pandemic, where reducing the exposure of patients and hospital staff to viral transmission is significant [56]. This also increases resources such as acute care beds, ventilators, advanced monitoring equipment and trained personnel that could be allocated to patients with severe illnesses.

The global pandemic has highlighted RA's ability to preserve postoperative immunological function, via the attenuation of the stress response and the associated effect on cellular and humoral immunity [62]. This may prove crucial when operating on a patient who may have COVID-19, diagnosed or undiagnosed, or who may be inadvertently exposed to it following surgery as the volatile gases used in GA are known to lower postoperative immunity [63]. Many routine operations such as orthopaedic emergencies, caesarean sections, ectopic pregnancies, strangulated hernias, appendectomies and urological operations are currently being performed under RA [3]. Furthermore, many cancer surgeries such as from simple to complex mastectomies and sentinel lymph node biopsies are also performed under RA.

\section{Conclusion}

The era of COVID-19 has changed innumerable aspects of the context in which medicine is practiced, introducing new considerations and shifting the relative importance of core priorities in the field of anaesthesiology no less than any other. This not only presents significant challenges to practitioners and researchers that must be overcome, but also provides an impetus for innovation. The increased emphasis on avoidance of viral transmission brings shortening hospital stays, as well as reducing respiratory and immunological impacts to the forefront of considerations to be made when determining optimal anaesthetic regimes for various surgical settings. Given the relative advantages of RA in these regards, we believe that the altered context of the COVID-19 pandemic creates both an imperative and an opportunity to devote more resources to the development and refinement of this subset of anaesthetic techniques.

Acknowledgements The authors would like to acknowledge Fatima Mansoor from The Kings School, Canterbury, UK, for her aid in language editing this manuscript.

Author Contribution All authors contributed to the manuscript idea and design. Material preparation, data collection and analysis were performed by all authors. The first draft of the manuscript was written by Raafay Mehmood and Ainsley McGuire, while all authors commented on previous versions of the manuscript. All authors read and approved the final manuscript.

Availability of Data and Material Not applicable.

Code Availability Not applicable.

\section{Declarations}

Ethics Approval Not applicable.

Consent to Participate Not applicable.

Consent for Publication Not applicable.

Conflict of Interest The authors declare no competing interests. 


\section{References}

1. Lie SA, Wong SW, Wong LT, et al (2020) Practical considerations for performing regional anesthesia: lessons learned from the COVID-19 pandemic. Can J Anesth

2. Mehmood R, Mansoor Z, Rashid F, Mehmood S. Journal of anesthesia and perioperative medicine opinion. J Anesth Perioper Care. 2020;1:1-2. https://doi.org/10.24015/JAPM.2017. 0055 .

3. Ashokka B, Chakraborty A, Subramania BJ, et al (2020) Reconfiguring the scope and practice of regional anesthesia in a pandemic: the COVID-19 perspective. Reg Anesth Pain Med. https://doi.org/10.1136/rapm-2020-101541

4. Pincus E. Regional anesthesia: an overview. AORN J. 2019;110:263-72. https://doi.org/10.1002/aorn.12781.

5. Nobre LV, Cunha GP, Sousa PCCB de, et al (2019) Peripheral nerve block and rebound pain: literature review. Brazilian J Anesthesiol (English Ed. https://doi.org/10.1016/j.bjane.2019.10.009

6. Gan TJ, Habib AS, Miller TE, et al (2014) Incidence, patient satisfaction, and perceptions of post-surgical pain: results from a US national survey. Curr Med Res Opin

7. Apfelbaum JL, Chen C, Mehta SS, Gan TJ (2003) Postoperative pain experience: results from a national survey suggest postoperative pain continues to be undermanaged. Anesth Analg https://doi.org/10.1213/01.ANE.0000068822.10113.9E

8. Gramke HF, De Rijke JM, Kleef M Van, et al (2009) Predictive factors of postoperative pain after day-case surgery. Clin J Pain. https://doi.org/10.1097/AJP.0b013e31819a6e34

9. Grant SA, Nielsen KC, Greengrass RA, et al (2001) Continuous peripheral nerve block for ambulatory surgery. Reg Anesth Pain Med. https://doi.org/10.1053/rapm.2001.22256

10. Pérez FAS, Sánchez GR (2011) Peripheral nerve blocks. Rev Colomb Anestesiol. https://doi.org/10.5554/rca.v39i3.88

11. Jin J, Wang G, Gong M, et al (2015) Retrospective comparison of the effects of epidural anesthesia versus peripheral nerve block on postoperative outcomes in elderly Chinese patients with femoral neck fractures. Clin Interv Aging. https://doi.org/ 10.2147/CIA.S87020

12. Brown DL, Ransom DM, Hall JA, et al (1995) Regional anesthesia and local anesthetic-induced systemic toxicity: seizure frequency and accompanying cardiovascular changes. Anesth Analg. https://doi.org/10.1097/00000539-199508000-00020

13. Borgeat A, Schäppi B, Biasca N, Gerber C (1997) Patient-controlled analgesia after major shoulder surgery: patient- controlled interscalene analgesia versus patient-controlled analgesia. Anesthesiology. https://doi.org/10.1097/00000542-199712000-00013

14. Moore JG, Ross SM, Williams BA (2013) Regional anesthesia and ambulatory surgery. Curr Opin Anaesthesiol

15. Singelyn FJ, Gouverneur JMA (1999) Postoperative analgesia after total hip arthroplasty: IV PCA with morphine, patientcontrolled epidural analgesia, or continuous "3-in-1" block?: A prospective evaluation by our acute pain service in more than 1,300 patients. J Clin Anesth. https://doi.org/10.1016/S09528180(99)00092-6

16. Yelnik AP, Hentzen C, Cuvillon P, et al (2019) French clinical guidelines for peripheral motor nerve blocks in a PRM setting. Ann Phys Rehabil Med

17. De Andrés J, Alonso-Iñigo JM, Sala-Blanch X, Reina MA (2005) Nerve stimulation in regional anaesthesia: theory and practice. Best Pract Res Clin Anaesthesiol.

18. Blumenfeld A, Ashkenazi A, Napchan U, et al (2013) Expert consensus recommendations for the performance of peripheral nerve blocks for headaches - a narrative review. Headache

19. Aguirre J, Del Moral A, Cobo I, et al (2012) The role of continuous peripheral nerve blocks. Anesthesiol. Res. Pract.
20. Nielsen KC, Steele SM (2002) Outcome after regional anaesthesia in the ambulatory setting - is it really worth it? Best Pract Res Clin Anaesthesiol. https://doi.org/10.1053/bean.2002.0244

21. Auroy Y, Narchi P, Messiah A, et al (1997) Serious complications related to regional anesthesia: results of a prospective survey in France. Anesthesiology. https://doi.org/10.1097/00000 542-199709000-00005

22. O'Donohoe PB, Pandit JJ. Physiology and pharmacology of spinal and epidural anaesthesia. Surg (United Kingdom). 2012;30:317-9.

23. Kompoliti K, Horn SS (2007) Drug-induced and iatrogenic neurological disorders. In: Textbook of Clinical Neurology: Third Edition. Elsevier Inc., pp 1285-1318

24. Visser WA, Lee RA, Gielen MJM. Factors affecting the distribution of neural blockade by local anesthetics in epidural anesthesia and a comparison of lumbar versus thoracic epidural anesthesia. Anesth Analg. 2008;107:708-21. https://doi.org/10.1213/ane. 0b013e31817e7065.

25. Rosenblatt WH SW (2013) 2013 Barash clinical anesthesia, 7th Ed.pdf

26. Moraca RJ, Sheldon DG, Thirlby RC. The role of epidural anesthesia and analgesia in surgical practice. Ann Surg. 2003;238:66373. https://doi.org/10.1097/01.sla.0000094300.36689.ad.

27. Gerheuser F, Roth A. Periduralanästhesie Anaesthesist. 2007;56:499-526. https://doi.org/10.1007/s00101-007-1181-1.

28. Vincent RD, Chestnut DH (1991) Which position is more comfortable for the parturient during identification of the epidural space? Int J Obstet Anesth. https://doi.org/10.1016/0959-289X(91) 90023-J

29. Todorov L, VadeBoncouer T. Etiology and use of the "hanging drop" technique: a review. Pain Res Treat. 2014;2014:1-10. https://doi.org/10.1155/2014/146750.

30. Brogly N, Guasch E, Alsina E, et al. Epidural space identification with loss of resistance technique for epidural analgesia during labor: a randomized controlled study using air or saline-new arguments for an old controversy. Anesth Analg. 2018;126:532-6. https://doi.org/10.1213/ANE.0000000000002593.

31. Antibas PL, do Nascimento Junior P, Braz LG, et al (2014) Air versus saline in the loss of resistance technique for identification of the epidural space. Cochrane Database Syst Rev CD008938. https://doi.org/10.1002/14651858.CD008938.pub2

32. Shenouda P. Assessing the superiority of saline versus air for use in the epidural loss of resistance technique: a literature review. Reg Anesth Pain Med. 2003;28:48-53. https://doi.org/10.1053/ rapm.2003.50009.

33. Segal S, Eappen S, Datta S. Superiority of multi-orifice over single-orifice epidural catheters for labor analgesia and cesarean delivery. J Clin Anesth. 1997;9:109-12. https://doi.org/10.1016/ S0952-8180(97)00232-8.

34. Conroy PH, Luyet C, McCartney CJ, McHardy PG. Real-time ultrasound-guided spinal anaesthesia: a prospective observational study of a new approach. Anesthesiol Res Pract. 2013;2013:1-7. https://doi.org/10.1155/2013/525818.

35. Di Cianni S, Rossi M, Casati A, et al (2008) Spinal anesthesia: An evergreen technique. Acta Biomed. l'Ateneo Parm.

36. Bolden N, Gebre E (2016) Accidental dural puncture management: 10-year experience at an academic tertiary care center. Reg Anesth Pain Med. https://doi.org/10.1097/AAP.0000000000 000339

37. Lee LA, Posner KL, Domino KB, et al (2004) Injuries associated with regional anesthesia in the 1980s and 1990s: a closed claims analysis. Anesthesiology. https://doi.org/10.1097/00000 542-200407000-00023

38. D'Angelo R, Smiley RM, Riley ET, Segal S (2014) Serious complications related to obstetric anesthesia. Anesthesiology. https:// doi.org/10.1097/aln.0000000000000253 
39. Davies JM, Posner KL, Lee LA, et al (2009) Liability associated with obstetric anesthesia: a closed claims analysis. Anesthesiology. https://doi.org/10.1097/ALN.0b013e318190e16a

40. Hotta K (2020) Regional anesthesia in the time of COVID19: a minireview. J Anesth. https://doi.org/10.1007/ s00540-020-02834-3

41. Kinsella SM, Girgirah K, Scrutton MJL. Rapid sequence spinal anaesthesia for category-1 urgency caesarean section: a case series. Anaesthesia. 2010;65:664-9. https://doi.org/10.1111/j. 1365-2044.2010.06368.x.

42. Varandas JS, Dias R, Mendes AB, et al (2020) New indication for an old anesthetic technique: could we consider now rapid sequence spinal anesthesia in a COVID-19 time? Reg Anesth Pain Med rapm-2020-101572. https://doi.org/10.1136/rapm-2020-101572

43. Zhong Q, Liu YY, Luo Q, et al. Spinal anaesthesia for patients with coronavirus disease 2019 and possible transmission rates in anaesthetists: retrospective, single-centre, observational cohort study. Br J Anaesth. 2020;124:670-5. https://doi.org/10.1016/j. bja.2020.03.007.

44. $\mathrm{Pu} \mathrm{X}$, Sun J. General anesthesia vs spinal anesthesia for patients undergoing total-hip arthroplasty. Medicine (Baltimore). 2019;98: e14925. https://doi.org/10.1097/MD.0000000000014925.

45. YaDeau JT, Fields KG, Kahn RL, et al. Readiness for discharge after foot and ankle surgery using peripheral nerve blocks. Anesth Analg. 2018;127:759-66. https://doi.org/10.1213/ANE.00000 00000003456.

46. Erdem VM, Donmez T, Uzman S, et al (2018) Spinal/epidural block as an alternative to general anesthesia for laparoscopic appendectomy: a prospective randomized clinical study. Videosurgery Other Miniinvasive Tech. https://doi.org/10.5114/wiitm. 2018.72684

47. Gulur P, Nishimori M, Ballantyne JC (2006) Regional anaesthesia versus general anaesthesia, morbidity and mortality. Best Pract. Res. Clin. Anaesthesiol.

48. Mason SE, Noel-Storr A, Ritchie CW (2010) The impact of general and regional anesthesia on the incidence of post-operative cognitive dysfunction and post-operative delirium: a systematic review with meta-analysis. J Alzheimer's Dis. https://doi.org/10. 3233/JAD-2010-101086

49. Curatolo M (2010) Adding regional analgesia to general anaesthesia: increase of risk or improved outcome? Eur. J. Anaesthesiol.

50. Hutton M, Brull R, Macfarlane AJR (2018) Regional anaesthesia and outcomes. BJA Educ.

51. Smith LM, Cozowicz C, Uda Y, et al. Neuraxial and combined neuraxial/general anesthesia compared to general anesthesia for major truncal and lower limb surgery. Anesth Analg. 2017;125:1931-45. https://doi.org/10.1213/ANE.0000000000 002069 .
52. Gan TJ, Diemunsch P, Habib AS, et al (2014) Consensus guidelines for the management of postoperative nausea and vomiting. Anesth. Analg.

53. Mashour GA, Pal D (2012) Interfaces of sleep and anesthesia. Anesthesiol. Clin.

54. Weinstein EJ, Levene JL, Cohen MS, et al (2018) Local anaesthetics and regional anaesthesia versus conventional analgesia for preventing persistent postoperative pain in adults and children. Cochrane Database Syst Rev. https://doi.org/10.1002/14651858. cd007105.pub4

55. Van Lier F, Van Der Geest PJ, Hoeks SE, et al (2011) Epidural analgesia is associated with improved health outcomes of surgical patients with chronic obstructive pulmonary disease. Anesthesiology. https://doi.org/10.1097/ALN.0b013e318224cc5c

56. Macfarlane AJR, Harrop-Griffiths W, Pawa A. Regional anaesthesia and COVID-19: first choice at last? Br J Anaesth. 2020;125:243-7. https://doi.org/10.1016/j.bja.2020.05.016.

57. Saraswat V (2015) Effects of anaesthesia techniques and drugs on pulmonary function. Indian J. Anaesth.

58. Guan W, Ni Z, Hu Y, et al (2020) Clinical characteristics of coronavirus disease 2019 in China. N Engl J Med. https://doi.org/10. 1056/nejmoa2002032

59. Horlocker TT, Vandermeuelen E, Kopp SL, et al (2018) Regional anesthesia in the patient receiving antithrombotic or thrombolytic therapy: American Society of Regional Anesthesia and Pain Medicine Evidence-Based Guidelines (Fourth Edition). Reg. Anesth. Pain Med.

60. El-Boghdadly K, Chin KJ, Chan VWS (2017) Phrenic nerve palsy and regional anesthesia for shoulder surgery: anatomical, physiologic, and clinical considerations. Anesthesiology

61. Schünemann HJ, Khabsa J, Solo K, et al (2020) Ventilation techniques and risk for transmission of coronavirus disease, including COVID-19: a living systematic review of multiple streams of evidence. Ann Intern Med. https://doi.org/10.7326/M20-2306

62. Šakić K, Žura M, Šakic L, et al (2009) Neuroimmunomodulation by regional and general anaesthesia. Period. Biol.

63. Wall T, Sherwin A, Ma D, Buggy DJ (2019) Influence of perioperative anaesthetic and analgesic interventions on oncological outcomes: a narrative review. Br. J. Anaesth.

Publisher's Note Springer Nature remains neutral with regard to jurisdictional claims in published maps and institutional affiliations. 